Check for updates

Cite this: Mater. Chem. Front., 2020, 4, 3678

Received 9th September 2020, Accepted 27th October 2020

DOI: 10.1039/d0qm00690d

rsc.li/frontiers-materials

\title{
Purity of organic semiconductors as a key factor for the performance of organic electronic devices $\dagger$
}

\author{
Cigdem Yumusak, Niyazi Serdar Sariciftci (D) and Mihai Irimia-Vladu*
}

\begin{abstract}
Organic semiconductors offer great promise through their ease of synthesis in a multitude of derivatives, their low temperature processability and their amenability for fabrication of flexible, stretchable and conformable, even imperceptible devices. Nevertheless, the low charge mobility of organic semiconductors remains one of the limiting factors in delivering high performance for organic electronic devices comparable to their inorganic counterparts. In this study, the effects of purification of eight different organic semiconductors (i.e. n-type, $\mathrm{p}$-type, and ambipolar) were determined by means of analyzing their performance improvement in organic field effect transistors. For this purpose, three purity grades of each organic semiconductor were investigated, and devices were fabricated in an identical fashion. It was found that temperature gradient sublimation improves considerably the quality of the organic semiconductors. The results presented here indicate that the purity of the organic semiconductor is a key parameter to be considered in order to achieve high performance for the field of organic field effect transistors.
\end{abstract}

\section{Introduction}

In the last two decades, a large effort has been directed towards integration of consumer electronics into emergent "embedded" electronics such as wearable/implantable electronics and internet of things, in other words industry 4.0. The most recent developments include on-tissue sensors, implantable and ingestible medical devices, environmental monitoring, and disposable plastic electronics such as smart food packaging, RFID tags, plastic cards, e-tickets, etc. ${ }^{1-15}$ All these devices require abundant and inexpensive materials and fabrication technologies supporting their sustainable development. Organic semiconductors are interesting through their synthetic versatility in high throughput at low cost of production. For achieving the coveted goal of "green" and sustainable electronics development, one can tailor and make these materials biocompatible and biodegradable. ${ }^{1,2}$ The large-scale electronic waste produced in the world is creating trouble in the supply chain of rare earth materials as well as plastic waste contamination in oceans, due to packaging materials in general. Both these unfortunate events can be faced successfully if we introduce biodegradable organic semiconductors, substrates and plastics. However, these materials

Linz Institute for Organic Solar Cells (LIOS), Physical Chemistry,

Johannes Kepler University Linz, Altenberger Straße 69, 4040 Linz, Austria

$\dagger$ This paper is dedicated to Prof. Fred Wudl on the occasion of his oncoming 80th birthday. have to be competitive to the performance requirements. Even though there are large areas of applications which do not need high frequency, high speed transistors and circuits, the mobility of organic semiconductor materials is still lower than their inorganic counterparts. Ultimately, the performance of the selected materials in the functional device will always be a competitive selection parameter parallel to their bio- and eco-friendliness. ${ }^{3}$

The design of new materials with required functional properties has always been a topical issue in Materials science. ${ }^{4-15}$ It includes both development of useful design rules and reproducible fabrication of new materials, which is particularly important for organic semiconductors to allow for a comprehensive understanding of their structure-property relationships. While studies have shown the great potential of organic materials in attaining excellent performance in optoelectronic devices, combining several functional properties in a single material able to match inorganic counterparts remains challenging. ${ }^{16-20}$ The organic field effect transistor (OFET) design is remaining still an active research area in organic electronics. ${ }^{19,21-26}$ In OFETs, every component layer, i.e. the substrate, dielectric, organic semiconductor, contact electrodes, and encapsulation layers, the choice of their materials as well as their geometries, is significant to the ultimate performance of the device.

Back in 2011 there were already crystalline OFETs with the mobility values exceeding those of amorphous silicon ${ }^{27-29}$ which was the main competitor in the past. ${ }^{21}$ Nevertheless, reaching 
values of field effect mobility in excess of $10 \mathrm{~cm}^{2} \mathrm{~V}^{-1} \mathrm{~s}^{-1}$ remains a tremendous challenge for the design and synthesis of novel organic semiconductors with increased functionalities, ${ }^{20,30}$ whereas attaining operation frequencies in advanced circuits design in the range of $\mathrm{GHz}$ seems possible, but rather difficult to materialize at the same time. ${ }^{31}$ We argue that all the benefits of inorganic semiconductors can be attributed to three major features of these materials: (i) their extremely high purity, (ii) their long-range order and (iii) their superior coupling through very strong (covalent) bonds. As a matter of fact, all the above beneficial characteristics that represent the cutting edge and the success of inorganic electronics are missing for the classic, i.e. weakly van der Waals bonded, organic semiconductors, where levels of purity of $99 \%$ or even lower are tacitly considered as acceptable for the fabrication of organic electronic devices. It is rather surprising that there is to the best of our knowledge no systematic study towards the elucidation of the role of purity of the organic semiconductors for the recorded performance of the fabricated thin film electronic devices. More often than not, organic electronic devices are fabricated with the "as received" materials carrying the declared purity by the chemical supplier, ${ }^{32}$ this event being obvious from the mere omission of the purification step of the organic semiconductor in the Experimental section of nearly all publications in the field. ${ }^{33-36}$ Nevertheless, when the effort of purification was invested, then the results improved impressively, with the recorded mobility of the organic semiconductor reaching record values in excess of $5 \mathrm{~cm}^{2} \mathrm{~V}^{-1} \mathrm{~s}^{-1}$ for single crystal based OFETs with rubrene. ${ }^{37}$ Importantly also, time of flight measurements at low temperature of ultra-pure oligomeric systems of organic semiconductors showed that mobilities of $10 \mathrm{~cm}^{2} \mathrm{~V}^{-1} \mathrm{~s}^{-1}$ at room temperature and of several hundred $\mathrm{cm}^{2} \mathrm{~V}^{-1} \mathrm{~s}^{-1}$ at low temperature are possible to achieve. ${ }^{38}$

In this work, we tackle the issue of the materials' purity and its influence on organic electronic devices systematically. We compared here the effect of different materials as well as different purification degrees respectively, on the performance of fabricated devices, to demonstrate systematically the influence of this purification versus performance relationship. We fabricated organic field effect transistors as a tool of evaluation of various purity grades of selected organic semiconductors. For this study a large pool of organic semiconductor materials, with n-, p-, and ambipolar-type charge transport were investigated. The selected semiconductors for this study were: fullerene $\left(\mathrm{C}_{60}\right)$, pentacene, copper phthalocyanine, indigo (vat blue 1), Tyrian purple, epindolidione, quinacridone and indanthrene blue RS (vat blue 4), the latter 5 molecules belonging to the group of hydrogen bonded (bio)-organic semiconductors. ${ }^{46}$ We used for the experimental, three grades of purity for each organic semiconductor and fabricated OFETs with the respective materials under identical thin film deposition conditions. The method of purification of the organic semiconductors investigated in this study was the train sublimation method, described previously in detail in other studies. ${ }^{37,39-44}$ Although the benefit of purification of organic materials for the improvement of their performance was addressed elsewhere, ${ }^{37,39-44}$ the quantitative results of various purification grades of organic semiconductors of this study clearly and systematically demonstrate that the mobility of charge carriers can be increased orders of magnitude alongside improving other device parameters.

\section{Experimental}

A photograph of one of the several vacuum sublimation ovens available in our laboratory is presented in Fig. 1. In the particular case displayed in Fig. 1, the sublimed material was quinacridone. The photograph shows by arrows the three important zones of the differential heating oven used for sublimation of the organic semiconductor. Several purification steps are necessary for obtaining a high-quality material, as will be demonstrated throughout this work.

For the scope of this study, each semiconductor material was analyzed in three purity grades: an unpurified grade (i.e. asreceived grade, denoted here as $0 \times$ ), a one-time purified grade (denoted as $1 \times$ in this study) and a three-times purified grade (denoted as $3 \times$ ). The only exception was Tyrian purple which was synthesized in-house through a previously reported method. ${ }^{45}$ In this particular case, the entire extracted material was purified scrupulously via column chromatography extraction, and this starting material for our study is designated here as $1 \times$. The successively purified grades employed in this study were $2 \times$ and $3 \times$ for Tyrian purple.

Sublimation purification of all the semiconductor materials used throughout this study was carried out using a quartz vacuum tube at a pressure below $1 \times 10^{-6}$ mbar. A series of borosilicate glass tubes were inserted into the quartz tube and served both to confine the source material and to serve as a vehicle of retrieving the sublimed material. Each material sublimed in a $24 \mathrm{~h}$ period at an individual temperature ranging from $250{ }^{\circ} \mathrm{C}$ to $400{ }^{\circ} \mathrm{C}$ and has been purified by up to three such successive temperature gradient sublimations. Each "as-received" semiconductor was subjected to three successive train sublimation steps in a horizontal vacuum oven, and the purest material was collected after each purification step. The materials collected after the one time and three times sublimation were the ones used in this study, alongside the "as-received" grade.

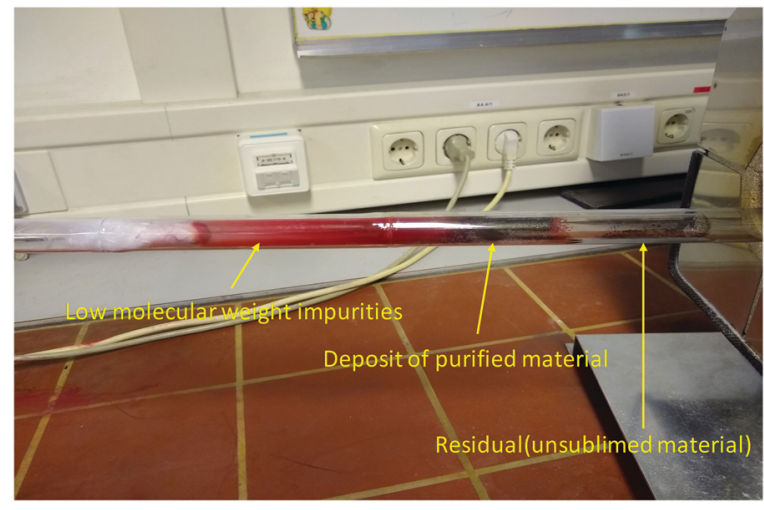

Fig. 1 Photograph of the train sublimation oven showing the sublimed material in the vacuum tube. 
Fullerene $\left(\mathrm{C}_{60}\right)$ was purchased from Aldrich, product number MKBR $8207 \mathrm{~V}$, having a labeled purity of 99.9\%. Pentacene, was purchased from Aldrich, product number P-1802. Copper phthalocyanine was purchased from Sigma, product number 252980. Quinacridone was purchased from TCI Europe, product number Q0057. Epindolidione was purchased from 1-Materials, product number HW1020P1. Indanthrene Blue RS was purchased from TCI Europe, product number I0429. Indigo was purchased from Aldrich, product number 229296, having a labeled purity of 95\%. Tyrian purple was synthesized "in-house" following the published synthetic routes. ${ }^{45}$

When fabricating the devices with each particular grade of purity, we obsessively obeyed all the 9 prerequisites of the reproducibility of results detailed in ref. 46. In short, all devices in this work were fabricated in the same Physical Vapor Deposition (PVD) System, using identical recipes of deposition (vacuum level, temperature ramp, deposition rate, etc.), and in an identical transistor geometry by employing the same shadow masks for the semiconductor structuring, as well as for the gate, source and drain electrode depositions, respectively. Importantly also, there was in this study no attempt made to optimize the results in the sense of adapting the rate of semiconductor deposition (either lower or faster), or play with the substrate heating in order to improve the performance; we used a standard recipe for all eight materials, and fabricated one batch only of 24 transistors for each purity grade of each material in the study. Importantly also, we used an identical dielectric for all materials in this study, namely a bi-layer of inorganic-organic dielectric consisting of electrochemically grown aluminum oxide (AlOx) of $34 \mathrm{~nm}$ thickness and a capping layer of tetratetracontane $\left(\mathrm{C}_{44} \mathrm{H}_{90}\right.$ or TTC as acronym $)$ of $20 \mathrm{~nm}$ thickness. The anodization method is a standard method employed in our laboratories and was previously described elsewhere. ${ }^{47,48}$ The thickness of the aluminum gate electrodes that were subjected to anodization was $100 \mathrm{~nm}$ for all the devices employed in this study. The respective layer of aluminum was evaporated at a very fast rate of 3-5 $\mathrm{nm} \mathrm{s}^{-1}$, which proved beneficial for generating a very smooth layer. ${ }^{49}$ We used for anodization in this work a recipe that employed passing to the sample to be anodized a step voltage up to a maximum of $20 \mathrm{~V}$, while maintaining in this process a steady current of $15 \mathrm{~mA}$. After reaching the $20 \mathrm{~V}$ compliance, the sample was allowed to slowly continue the anodization until the final current read $4.5 \mu \mathrm{A}$, which translated to a typical waiting time of about 6-7 min per sample, in our gate geometry. Using this method, as a rule of thumb, one obtains a typical thickness of the anodized layer as

$$
d=a\left(V-V_{\mathrm{ox}}\right),
$$

where $a$ is the oxide forming factor, which has a value of 1.6 for aluminum; $V$ is the applied maximum voltage of anodization and $V_{\text {ox }}$ is the voltage necessary to generate the native oxide (i.e. the layer that forms naturally when aluminum is exposed to ambient air), and its value is $V_{\text {ox }}=\sim-1.35 \mathrm{~V}$ for aluminum. ${ }^{50}$ The measured bi-layer capacitance in a Novocontrol Impedance Analyzer instrument matched very well the calculated capacitance of the combo layer of $\sim 34 \mathrm{~nm} \mathrm{AlOx}(\sim 32 \mathrm{~nm}$ electrochemically grown plus $\sim 2 \mathrm{~nm}$ native) and $20 \mathrm{~nm}$ TTC, and the respective value of $77.77 \mathrm{nF} \mathrm{cm} \mathrm{cm}^{-2}$ was used therefore for all the transistor related calculations in this work. For all transistors in this work, we evaporated the $20 \mathrm{~nm}$ tetratetracontane aliphatic capping layer at a rate of deposition between 0.7 and $1 \mathrm{~A} \mathrm{~s}^{-1}$, and performed an in situ annealing at $65{ }^{\circ} \mathrm{C}$ for 30 min inside the PVD system prior to the semiconductor deposition. This annealing step proved necessary for smoothening the dielectric and allow for a better coverage of the aluminum oxide layer, as we demonstrated and reported before for several other dielectrics. ${ }^{51-54}$ All the semiconductors were evaporated with an identical recipe, at a rate of $0.2-0.3 \AA \mathrm{s}^{-1}$ to account for a final thickness of $60 \mathrm{~nm}$. Top contact electrodes (aluminum in the case of the devices with $\mathrm{C}_{60}$ and gold for all the others) were deposited at various deposition rates: $0.1 \mathrm{~nm} \mathrm{~s}^{-1}$ for the devices with $\mathrm{C}_{60}$ and pentacene, and between 0.7 and $1 \mathrm{~nm} \mathrm{~s}^{-1}$ for the rest of the semiconductors in this study. In this respect we showed previously that faster deposition rates of gold contact electrodes are desirable for the hydrogen bonded organic semiconductors. ${ }^{46,52,55-58}$ All the measurements of the fabricated OFETs were carried out with the aid of a probe station situated in a glove box under a nitrogen atmosphere.

\section{Results}

\subsection{Organic field effect transistors}

The typical device structure utilized in our laboratory employs a staggered bottom gate top contact structure, like the one presented in Fig. 2, with the component layers marked on the figure.

The investigated key parameters of organic field-effect transistors are: the operating voltage, the semiconductor charge carrier mobility, the on/off current ratio, the subthreshold swing and finally the threshold voltage and its stability during operation. ${ }^{59}$ Fig. 3 presents an example of a field effect transistor of high mobility fabricated previously in our laboratories. ${ }^{60}$ It is a device fabricated in a bottom gate-top contact geometry like the one schematically presented in Fig. 2, with adenine capped aluminum oxide as the dielectric layer and fullerene $\mathrm{C}_{60}$ as the organic semiconductor. The graph in Fig. 3(a) shows the recorded transfer characteristics of the

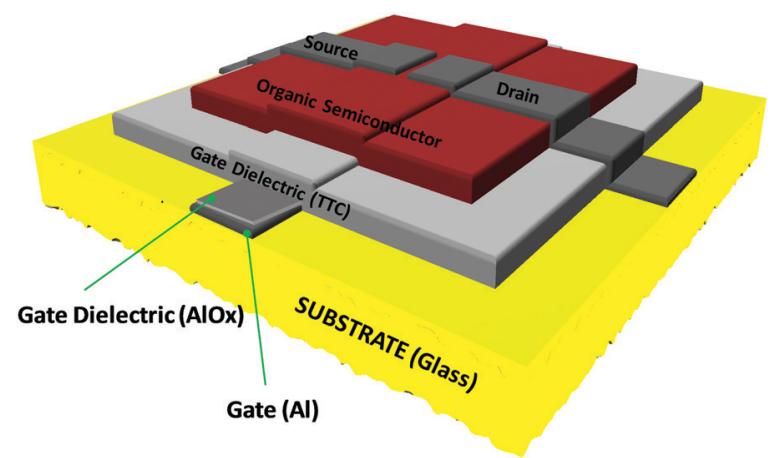

Fig. 2 OFET device architecture in the bottom gate-top contact configuration. 
a)

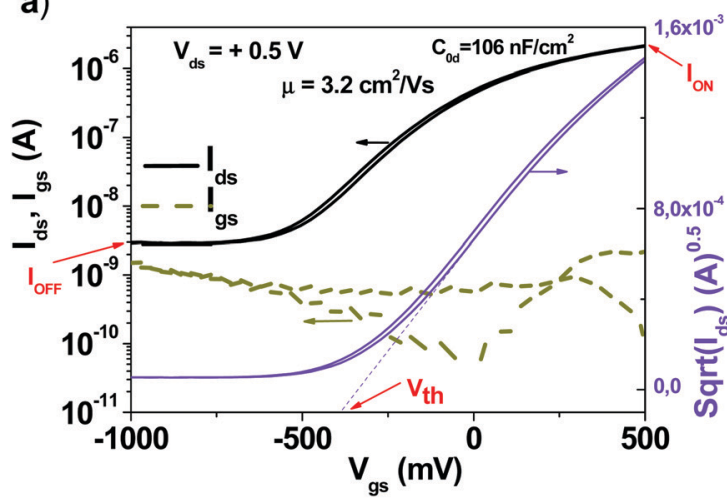

b)

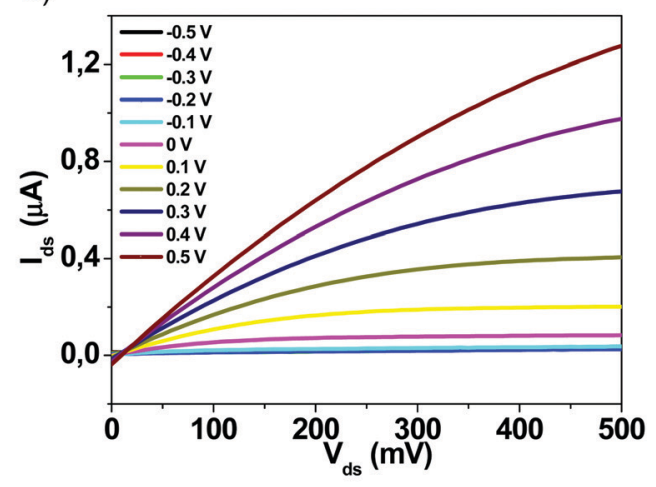

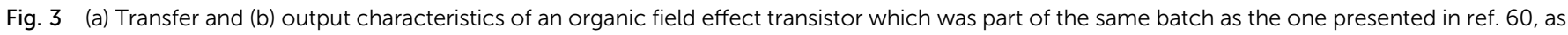

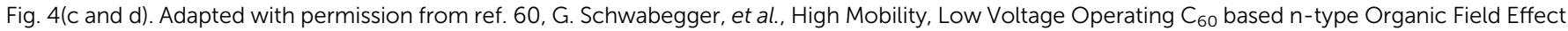
Transistors, Synth. Met., 2011, 161, 2058-2062.

OFET, i.e. the measured $I_{\mathrm{ds}} v s$. $V_{\mathrm{gs}}$ at constant $V_{\mathrm{ds}}$ voltage, while the one in Fig. 3(b) shows the corresponding output characteristics of the OFET, i.e. the curves measured as $I_{\mathrm{ds}} v s$. $V_{\mathrm{ds}}$ at constant $V_{\text {gs }}$ voltages. The important parameters of the device, the ON/OFF ratio and the threshold voltage $\left(V_{\text {th }}\right)$ are displayed in the transfer characteristics graph. The average charge mobility of the organic semiconductor in the fabricated batch of which the OFET displayed in Fig. 3 belongs was $3.2 \mathrm{~cm}^{2} \mathrm{~V}^{-1} \mathrm{~s}^{-1}$.

The ON/OFF ratio is the ratio between the current in no conduction mode (marked as $I_{\mathrm{OFF}}$ on the graph) and the maximum current attained in the operation (conduction mode, marked as $I_{\mathrm{ON}}$ on the graph). In the particular example of the device presented in Fig. 3, this ratio is coming close to 1000, or $10^{3}$. The threshold voltage is determined as the intercept on the horizontal axis of the tangent drawn to the linear part of the square root of the $I_{\mathrm{ds}} v s$. $V_{\mathrm{gs}}$ line in the saturation regime of the transistor (marked as $V_{\text {th }}$ on the graph). For mobility calculations when a straight line is not an accurate fit to the $\operatorname{sqrt}\left(I_{\mathrm{ds}}\right)$ we fitted the line at higher gate voltages. We determined the start and end points of the subthreshold swing from the best straight line fit to a gate voltage interval on the $\log \left(I_{\mathrm{ds}}\right)$ vs. $V_{\text {gs }}$ plot for lower gate voltages than the threshold voltage as discussed in the review paper of Newman et al. (ref. 59).

The field effect mobility of the semiconductor in the saturation regime is extracted from the equation: ${ }^{59}$

$$
I_{\mathrm{ds}, \mathrm{sat}}=(W / 2 L) C_{\mathrm{od}} \mu_{\mathrm{sat}}\left(V_{\mathrm{gs}}-V_{\mathrm{th}}\right)^{2},
$$

whereas the subthreshold swing $\left(S_{\mathrm{sw}}\right)$ is the inverse of the subthreshold slope $\left(S_{\mathrm{sl}}\right)$, and can be extracted from the equation: ${ }^{59}$

$$
S_{\mathrm{sw}}=1 / S_{\mathrm{sl}}=\mathrm{d} V_{\mathrm{gs}} / \mathrm{d}\left(\log I_{\mathrm{ds}}\right),
$$

while the normalized subthreshold swing $\left(S_{\mathrm{sn}}\right)$ is given by the equation:

$$
S_{\mathrm{sn}}=S_{\mathrm{sw}} \times C_{\mathrm{od}}
$$

In the above equations $I_{\mathrm{ds} \text {,sat }}$ represents the drain current in the saturation regime, $W$ and $L$ are the geometric factors of the channel (length, $L$ and width, $W$, respectively), $C_{0 \mathrm{~d}}$ is the specific capacitance of the dielectric, $\mu_{\text {sat }}$ is the field effect mobility of the organic semiconductor in the saturation regime, $V_{\mathrm{gs}}$ and $V_{\mathrm{th}}$ are the applied gate voltage and the threshold voltage, respectively, and $S_{\mathrm{sw}}$ is the subthreshold swing, expressed in volts per decade of current (i.e. $\mathrm{V} \operatorname{dec}^{-1}$ ). The subthreshold swing indicates how fast the OFET device switches form the OFF to the ON state. It is worth mentioning that the normalized subthreshold swing is a more convenient method to compare the performance of organic semiconductors in OFET devices, when deposited on various dielectrics or on dielectrics of different thicknesses. ${ }^{59}$ A large value of the subthreshold swing indicates a large concentration of shallow traps, i.e. a slow and non-sharp turn-on region. Given the fact that we have in this study the same dielectric but different purity grades of organic semiconductors, the normalized subthreshold swing values are meaningful as a method of comparison of the performance of dielectric-semiconductor interface within the three purity grades of each semiconductor material.

The schematic of the semiconductor molecules employed in this work is presented in Fig. 4.

\subsection{Fullerene, $\mathrm{C}_{60}$}

The schematic of the $\mathrm{C}_{60}$ molecule is presented in Fig. 4 . We fabricated devices with fullerene $\left(\mathrm{C}_{60}\right)$ and compared the three purity grades mentioned above, i.e. $0 \times, 1 \times$ and $3 \times$ in OFET devices. It is interesting to note that although the original $(0 \times)$ material had a declared purity of $99.9 \%$ according to the label on the material package of the chemical supplier (Aldrich), the average mobility obtained for the 24 transistors fabricated was in the range of $5 \times 10^{-5} \mathrm{~cm}^{2} \mathrm{~V}^{-1} \mathrm{~s}^{-1}$ (black line in Fig. 5).

The one-time sublimed material showed a more decent performance, with mobility values already in the range of $1 \times 10^{-2} \mathrm{~cm}^{2} \mathrm{~V}^{-1} \mathrm{~s}^{-1}$. One sublimation of fullerene resulted therefore in a 200 -fold increase in mobility, while three successive purification steps on the other hand counted for another 4-fold increase in the organic semiconductor mobility compared to the one-time purified grade (in total almost three orders of 


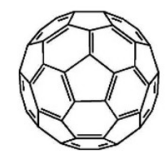<smiles>c1ccc2cc3cc4cc5ccccc5cc4cc3cc2c1</smiles>

$\mathrm{C}_{60}$

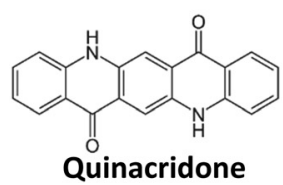

Pentacene

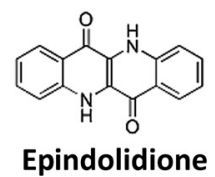

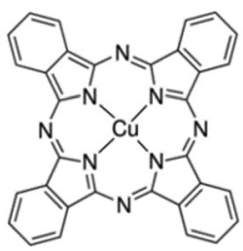

Copper Phthalocyanine

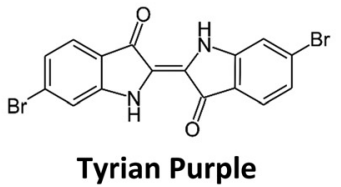

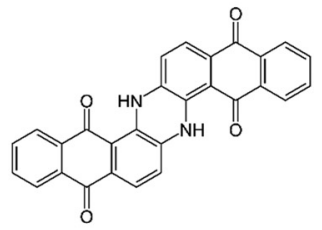

Indanthrene Blue RS

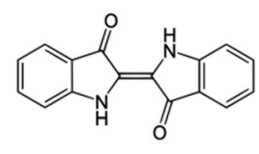

Indigo

Fig. 4 Schematic of the molecules employed in this study.
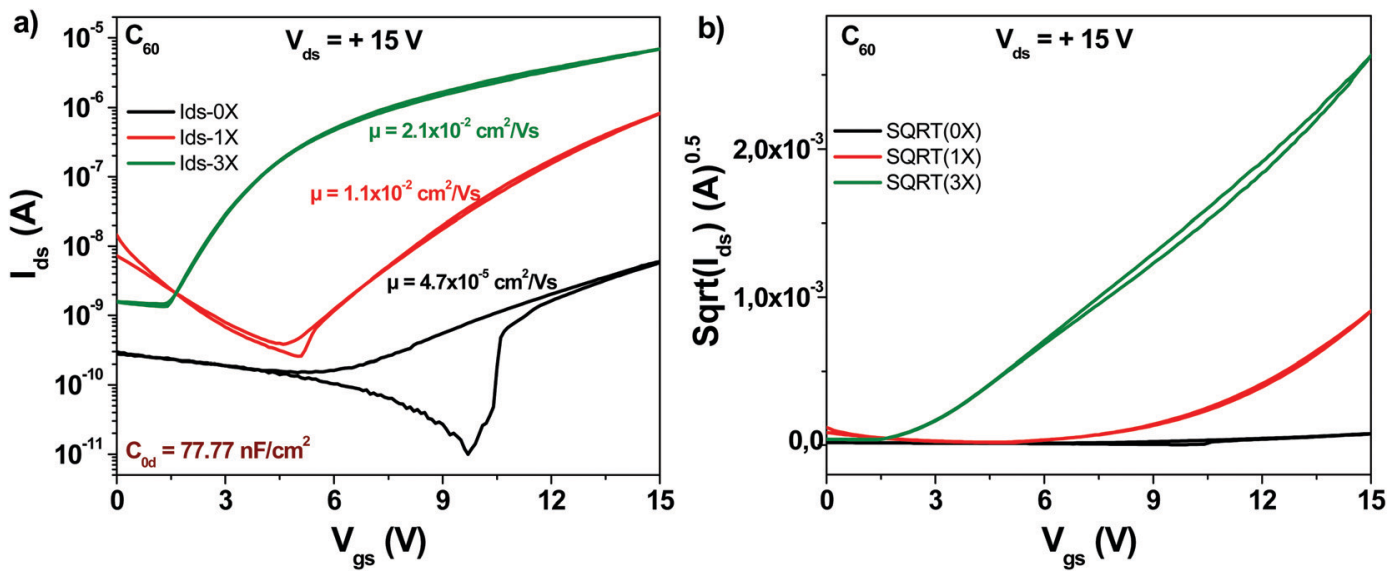

Fig. 5 (a) Comparison of OFET devices with three different grades of fullerene $\left(C_{60}\right)$ semiconductor purity: unpurified $(0 \times$ in graph legend), one time purified ( $1 \times$ in graph legend) and three times purified respectively $\left(3 \times\right.$ in graph legend); (b) the sqrt $\left(I_{\mathrm{ds}}\right) v \mathrm{~s}$. $V_{\mathrm{gs}}$ of the three purity grades presented in panel (a).

magnitude compared to the unpurified grade). It is interesting to note also that three steps of material purification decreased the subthreshold swing of the transistor from 4.8 to $1.1 \mathrm{~V} \mathrm{dec}^{-1}$. Full details over the transistor's parameters (subthreshold swing, subthreshold slope, ON/OFF ratio and normalized subthreshold swing) are offered in Table 1.

\subsection{Pentacene}

The schematic of the pentacene molecule is displayed in Fig. 4 . The OFET transistor characteristics are presented in Fig. 6 . Different than $\mathrm{C}_{60}$ though, the one-time purified grade does not show a major improvement in transistor mobility as compared to the unpurified grade, i.e. only a 4 -fold increase, from $1.9 \times$ $10^{-4}$ to $7.5 \times 10^{-4} \mathrm{~cm}^{2} \mathrm{~V}^{-1} \mathrm{~s}^{-1}$. Nevertheless, the three times purified grade of pentacene shows a marked increase in mobility that reached $2 \times 10^{-2} \mathrm{~cm}^{2} \mathrm{~V}^{-1} \mathrm{~s}^{-1}$ (100-fold compared to the unpurified grade). At the same time, the three times purified material showed a significant decrease of the subthreshold swing compared to the unpurified material, i.e. from 2.5 to $1.4 \mathrm{~V} \mathrm{dec}^{-1}$, with full details of the transistor measurements offered in Table 1.

\subsection{Copper phthalocyanine}

The schematic of the copper phthalocyanine molecule is displayed in Fig. 4. OFET devices employing the "as received" grade showed as in the case of fullerene and pentacene a modest mobility value, in the range of $2 \times 10^{-5} \mathrm{~cm}^{2} \mathrm{~V}^{-1} \mathrm{~s}^{-1}$ (see Fig. 7). However, one single step of purification resulted in a charge mobility increase of 35 -fold, to $9 \times 10^{-4} \mathrm{~cm}^{2} \mathrm{~V}^{-1} \mathrm{~s}^{-1}$. Two more purification steps made possible attaining a mobility of $2 \times 10^{-3} \mathrm{~cm}^{2} \mathrm{~V}^{-1} \mathrm{~s}^{-1}$, in other words an 80-fold increase from the original, unpurified material. Notably, the subthreshold swing decreased from $4.1 \mathrm{~V} \mathrm{dec}^{-1}$ to $1.6 \mathrm{~V} \mathrm{dec}^{-1}$ after three sublimation steps. Full details of the subthreshold parameters as well as the ON/OFF ratios and threshold voltages for the OFETs featuring the three purity grades of copper phthalocyanine OFETs are presented in Table 1.

\subsection{Indanthrene blue RS}

Indanthrene blue RS (vat blue 4) employed in this study has the chemical structure presented in Fig. 4. Different from the previously described materials in this study, indanthrene blue 
Table 1 OFET values for the semiconductors employed in this work. Legend acronyms are: $\mathrm{e}^{-}-$electron; $\mathrm{h}^{+}-$hole; $I_{\text {on }} / I_{\text {off }}-$ on-off ratio of the drainsource current; $S_{s w}-$ subthreshold swing; $S_{n}$ - normalized subthreshold swing; $V_{t h}$ - threshold voltage. The mobility values represent average values of 24 transistors in the respective batch. The departure from the average value of the batch is no greater than $10 \%$ for all transistors

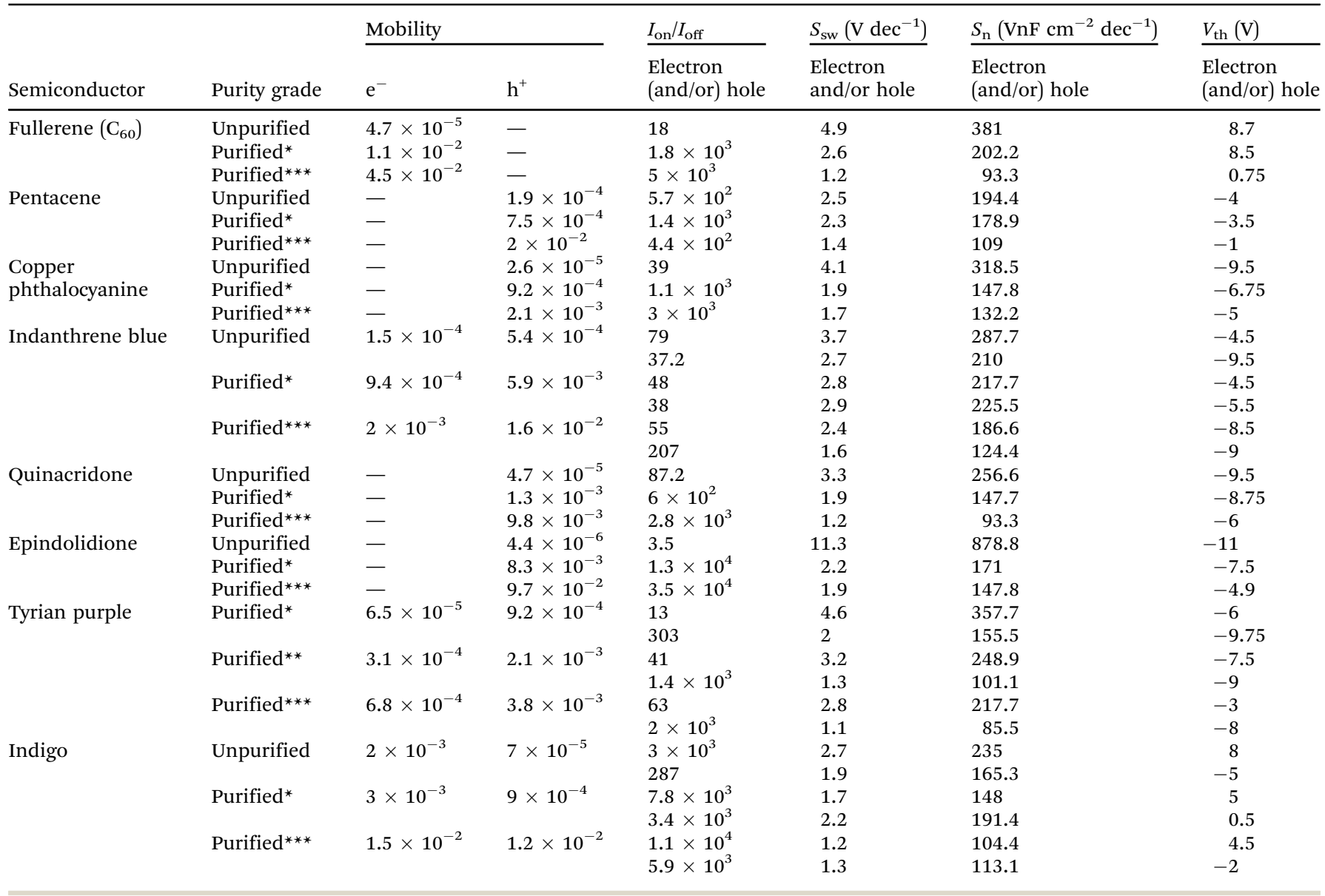
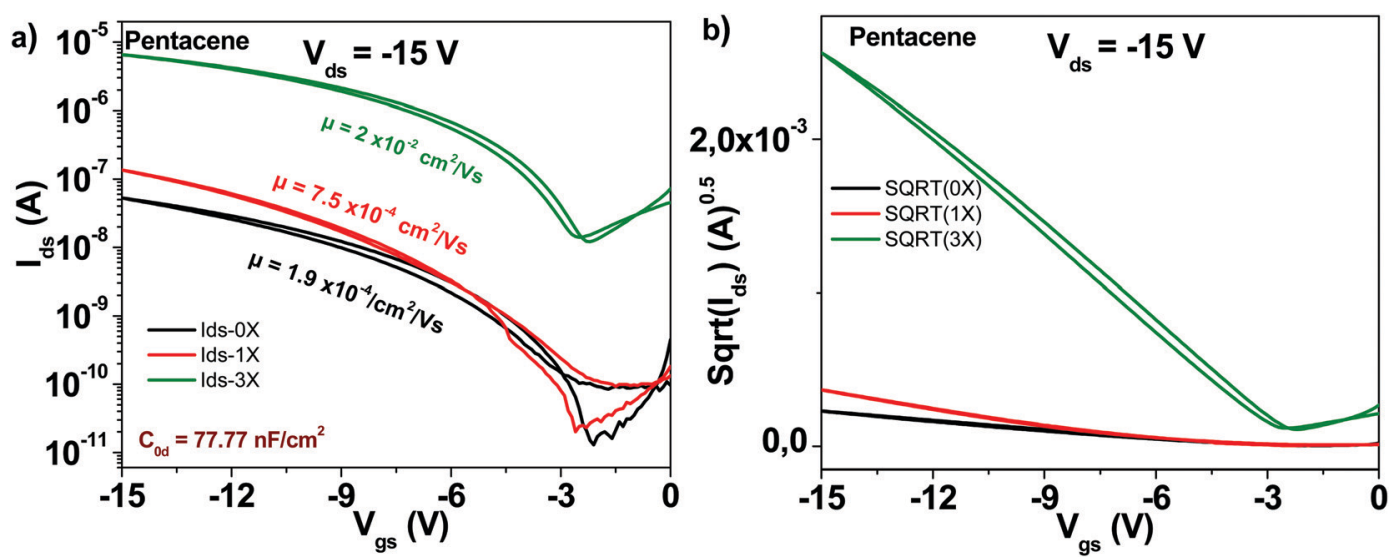

Fig. 6 (a) Comparison of OFET devices with three different purity grades of pentacene semiconductor: unpurified $(0 \times$ in graph legend), one time purified ( $1 \times$ in graph legend) and three times purified respectively $\left(3 \times\right.$ in graph legend); (b) the sqrt $\left(I_{d s}\right) v s . V_{g s}$ of the three purity grades presented in panel (a).

RS is an ambipolar semiconductor when it is deposited on top of aliphatic dielectrics (TTC in this study). Transistor characteristic results of OFETs with indanthrene blue RS semiconductor are displayed in Fig. 8. As in the case of other organic semiconductors employed in this study, three steps of sublimation proved beneficial for the generation of superior transistor characteristics. Starting from an unpurified material with electron and hole mobilities in the range of $\sim 10^{-4} \mathrm{~cm}^{2} \mathrm{~V}^{-1} \mathrm{~s}^{-1}$, three purification steps increased the respective values to $\sim 2 \times 10^{-2} \mathrm{~cm}^{2} \mathrm{~V}^{-1} \mathrm{~s}^{-1}$ for the hole channel and $2 \times 10^{-3} \mathrm{~cm}^{2} \mathrm{~V}^{-1} \mathrm{~s}^{-1}$ for the electron channel, respectively. Notably, the subthreshold swing also decreased from 3.6 to 

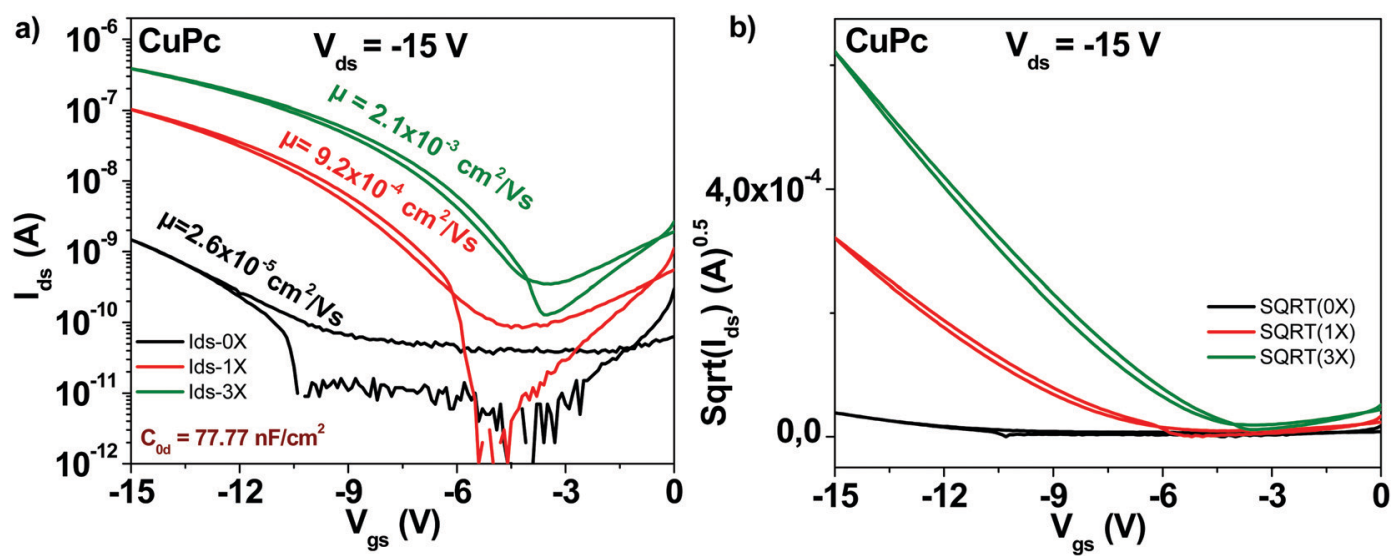

Fig. 7 (a) Comparison of OFET devices with three different purity grades of copper phthalocyanine semiconductor: unpurified ( $0 \times$ in graph legend), one time purified ( $1 \times$ in graph legend) and three times purified, respectively $\left(3 \times\right.$ in graph legend); (b) the sqrt $\left(I_{\mathrm{ds}}\right) v s$. $V_{\mathrm{gs}}$ of the three purity grades presented in panel (a).
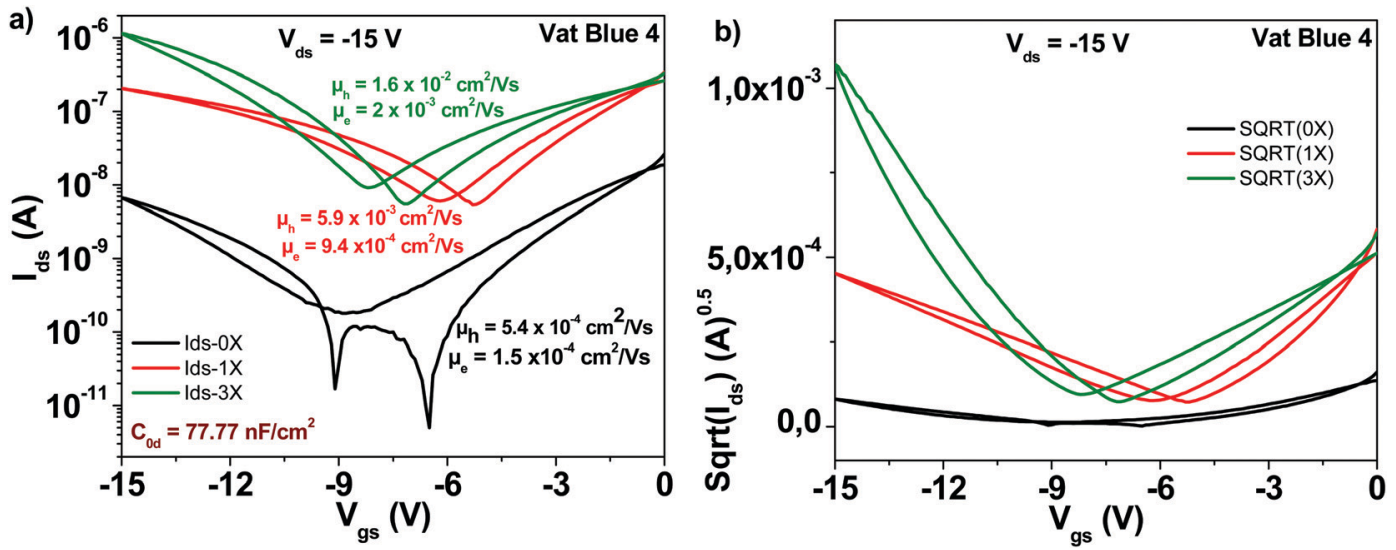

Fig. 8 (a) Comparison of OFET devices with three different purity grades of vat blue 4 semiconductor: unpurified ( $0 \times$ in graph legend), one time purified $\left(1 \times\right.$ in graph legend) and three times purified, respectively $\left(3 \times\right.$ in graph legend); (b) the $\operatorname{sqr}\left(I_{\mathrm{ds}}\right) v s$. $V_{\mathrm{gs}}$ of the three purity grades presented in panel (a).

$2.4 \mathrm{~V} \mathrm{dec}^{-1}$ for the electron channel and from 2.7 to $1.6 \mathrm{~V} \mathrm{dec}^{-1}$ for the hole channel, and full details of the indanthrene blue RS based OFET performance are presented in Table 1.

\subsection{Quinacridone}

The chemical structure of the quinacridone molecule is displayed in Fig. 4. In line with the findings for the previously described semiconductors, also quinacridone displayed a tremendous increase in mobility and decrease of subthreshold swing after three purification steps, see Fig. 9. Starting from a field effect mobility of $\sim 5 \times 10^{-5} \mathrm{~cm}^{2} \mathrm{~V}^{-1} \mathrm{~s}^{-1}$ for an unpurified, "as received" grade, quinacridone's field effect mobility reached $\sim 1 \times 10^{-2} \mathrm{~cm}^{2} \mathrm{~V}^{-1} \mathrm{~s}^{-1}$ for the three times purified grade. At the same time, the subthreshold swing decreased from 3.2 to $1.2 \mathrm{~V} \mathrm{dec}^{-1}$ respectively, accompanied by an increase in the ON/OFF ratio from 87 to 2800 . Full details of the transistor characteristic parameters are offered in Table 1.

\subsection{Epindolidione}

The chemical structure of the epindolidione molecule is displayed in Fig. 4. Among the studied materials in this work, epindolidione was the one that produced the most remarkable improvement after being subjected to three steps of purification. Indeed, starting from very low field effect mobility of the "as received" material of $4.4 \times 10^{-6} \mathrm{~cm}^{2} \mathrm{~V}^{-1} \mathrm{~s}^{-1}$, epindolidione based OFETs almost reached the mobility of $0.1 \mathrm{~cm}^{2} \mathrm{~V}^{-1} \mathrm{~s}^{-1}$ after being subjected to three steps of scrupulous purification, or in other words an improvement in excess of 22000 . This tremendous mobility improvement was accompanied by a decrease of the subthreshold swing from 11.3 to $1.9 \mathrm{~V} \mathrm{dec}^{-1}$ and an increase of the ON/OFF ratio from 3.5 to $3.5 \times 10^{4}$, literally a 10000 -fold improvement (Fig. 10). Full details of the transistor characteristics for epindolidione based OFETs with the three grades of analyzed purity are offered in Table 1.

\subsection{Tyrian purple}

A schematic of the Tyrian purple molecule $\left(6,6^{\prime}\right.$-Br-Indigo) is presented in Fig. 4 . The results with OFETs carrying the three grades of pure Tyrian purple are displayed in Fig. 11. Given the good purity of Tyrian purple to start with $(1 \times)$ in this study, it makes full sense that this material displayed the lowest improvement among all the other organic semiconductors 

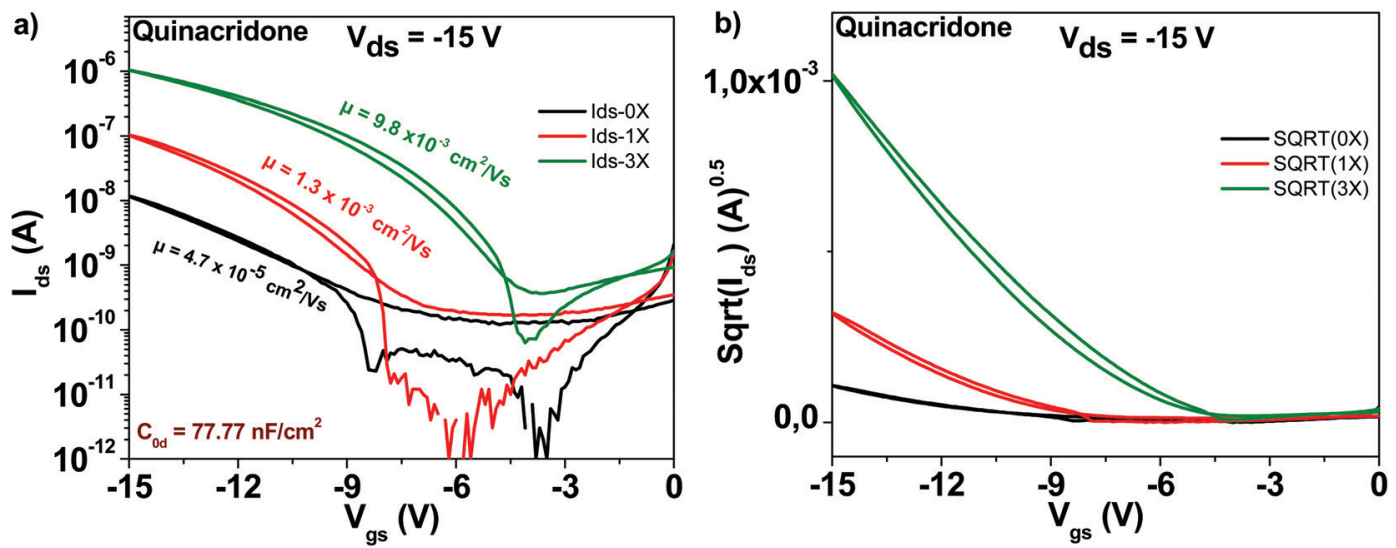

Fig. 9 (a) Comparison of OFET devices with three different purity grades of quinacridone semiconductor: unpurified $(0 \times$ in graph legend), one time purified $\left(1 \times\right.$ in graph legend) and three times purified, respectively $\left(3 \times\right.$ in graph legend); (b) the sqrt $\left(I_{\mathrm{ds}}\right) v s . V_{\mathrm{gs}}$ of the three purity grades presented in panel (a).
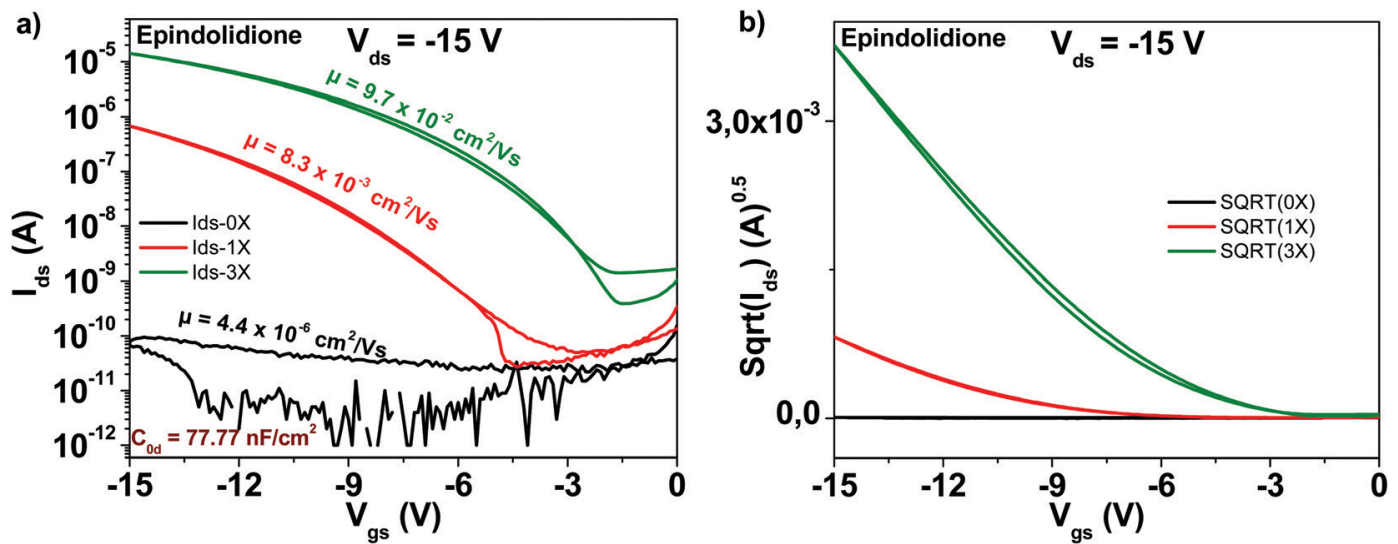

Fig. 10 (a) Comparison of OFET devices with three different purity grades of epindolidione semiconductor: unpurified (0x in graph legend), one time purified $\left(1 \times\right.$ in graph legend) and three times purified, respectively $\left(3 \times\right.$ in graph legend); (b) the sqrt $\left(I_{\mathrm{ds}}\right) v s$. $V_{\mathrm{gs}}$ of the three purity grades presented in panel (a).

analyzed in this study. After three sublimation steps, the electron channel improved by a little bit over one order of magnitude in field effect mobility (i.e. $\sim 6 \times 10^{-5}$ to $\sim 6 \times$ $\left.10^{-4} \mathrm{~cm}^{2} \mathrm{~V}^{-1} \mathrm{~s}^{-1}\right)$, whereas the hole channel improved by a factor of 4 only (i.e. from $\sim 9 \times 10^{-4}$ to $\sim 4 \times 10^{-3} \mathrm{~cm}^{2} \mathrm{~V}^{-1} \mathrm{~s}^{-1}$ ). However, the successive purification steps did indeed improve the material performance, a fact that is obvious in the obtained transistor parameters displayed in Table 1, i.e. the subthreshold swing decreased for the electron channel from 4.6 to $2.8 \mathrm{~V} \mathrm{dec}^{-1}$, while for the hole channel from 2 to $1 \mathrm{~V} \mathrm{dec}^{-1}$, when comparing the $1 \times$ and $3 \times$ purity grades.

\subsection{Indigo}

A schematic of an indigo molecule is presented in Fig. 4. Indigo employed in this study was reported in our previous publication (ref. 46). In this work we present in addition, the tabulated values of the transistor parameters. It is obvious that performing three rounds of purification not only increases the mobility of the semiconductor but also decreases the subthreshold swing from $\sim 2.7$ to $1.2 \mathrm{~V} \mathrm{dec}^{-1}$ for the electron channel and from $\sim 1.9$ to
$1.3 \mathrm{~V} \mathrm{dec}^{-1}$ for the hole channel respectively (see Fig. 12). Full details of the recorded/calculated values of the indigo transistor performance are presented in Table 1 .

\section{Discussion and conclusions}

We showed here that scrupulous purification of the organic semiconductor is a critical parameter in improving the performance of the organic field effect transistors. We should point out here that each semiconductor material has itself a designated (specific) purification recipe, that differ from one another in the temperature ramping, the differential heating of the vacuum tube, and mostly the flow rate of the carrier gas and its type (Ar, $\mathrm{N}_{2}$, or even no carrier gas, etc.). All these factors are material specific and cannot be generalized. In this work we demonstrated that by pursuing several purification steps starting from the pristine material, all the transistor parameters (apart from for some few exceptions, the threshold voltage, $V_{\text {th }}$ ) consistently improved alongside the purity of the organic semiconductors. In several cases (see Table 1) the mobility 

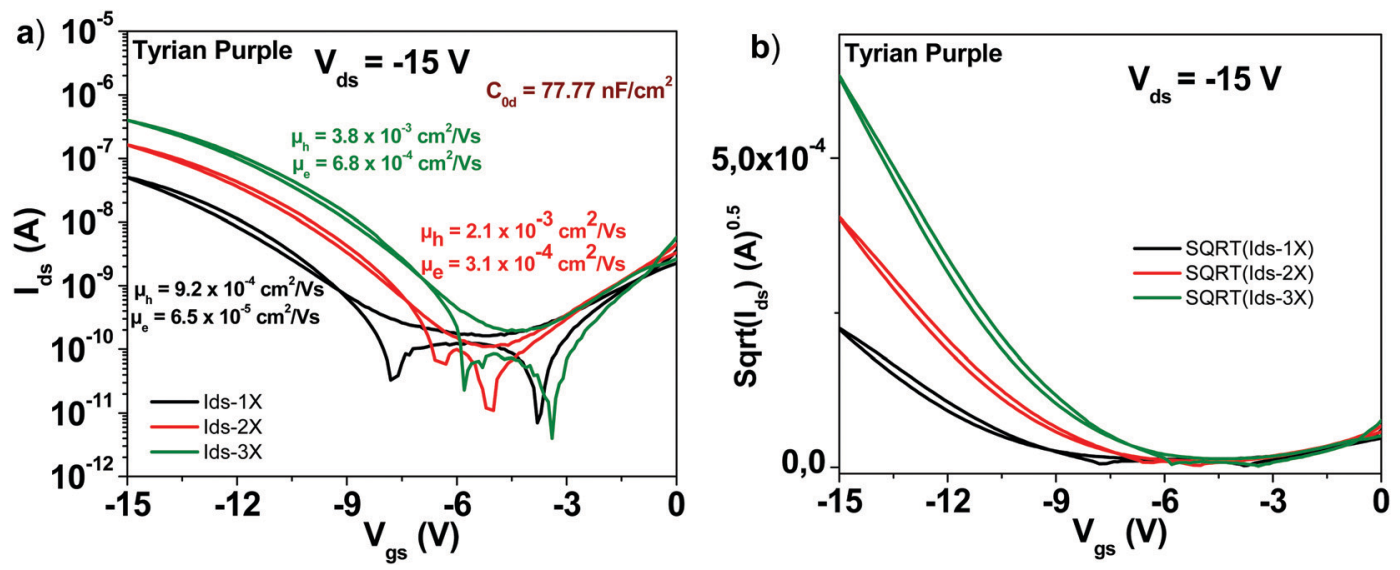

Fig. 11 (a) Comparison of OFET devices with three different purity grades of Tyrian purple semiconductor: one-time purified ( $1 \times$ in graph legend), two times purified ( $2 \times$ in graph legend) and three times purified, respectively $\left(3 \times\right.$ in graph legend); (b) the sqrt $\left(l_{\mathrm{ds}}\right) v s$. $V_{\mathrm{gs}}$ of the three purity grades presented in panel (a).
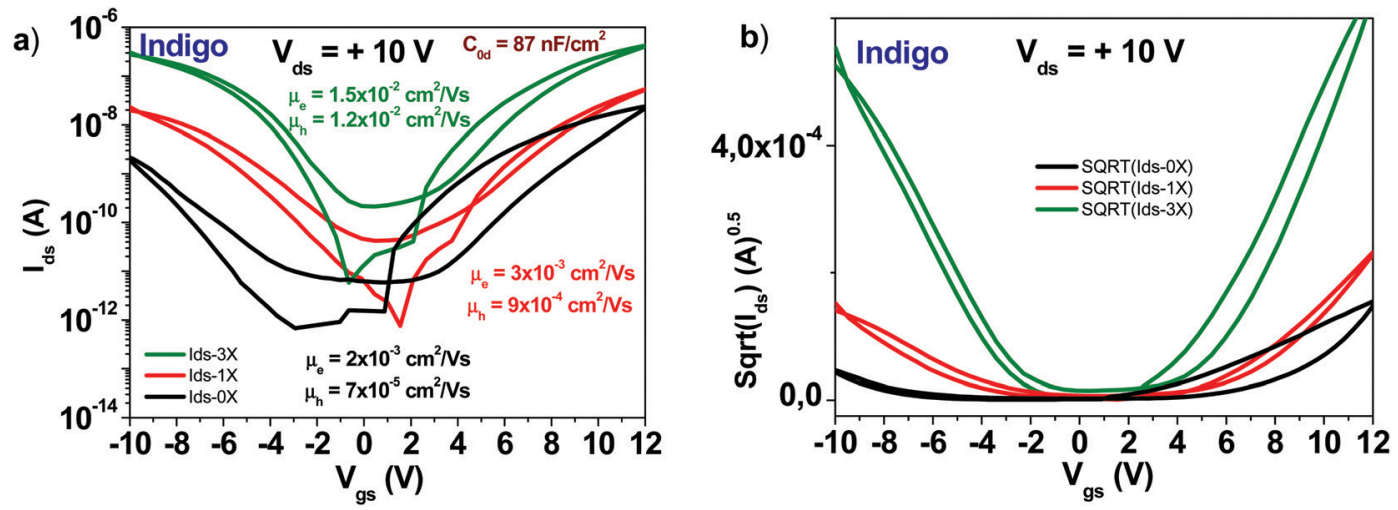

Fig. 12 (a) Comparison of OFET devices with three different purity grades of indigo semiconductor: an unpurified grade $(0 \times$ in graph legend), a onetime purified grade ( $1 \times$ in graph legend) and a three times purified grade, respectively $(3 \times$ in graph legend). The specific capacitance of Indigo OFETs is $87 \mathrm{nF} \mathrm{cm}{ }^{-2}$; (b) the sqrt $\left(I_{\mathrm{ds}}\right)$ vs. $V_{\mathrm{gs}}$ of the three purity grades presented in panel (a). Adapted with permission from ref. 46, M. Irimia-Vladu, et al., Chem. Mater., 2019, 31(17), 6315-6346.

improvement surpassed three orders of magnitude, whereas the subthreshold swing decreased to values close to $1 \mathrm{~V} \mathrm{dec}^{-1}$.

Inspecting Fig. 5-12 presenting transistor measurements in this manuscript, as well as Fig. 3 which displayed a high purity $\mathrm{C}_{60}$-based OFET scrupulously optimized for high mobility values, it is interesting to note that for all the analyzed materials, always the highly purified semiconductor grade displays a much higher OFF level on the transfer characteristics as compared to the pristine, non-purified material. This is in a way counterintuitive, since a higher OFF level of the drain current is usually given by impurities (high doping levels). We consider that the change in morphology of the deposited semiconductor has also a significant influence when comparing various purity grades; and also the fact that we measured all the transistors in the accumulation regime (from zero $V_{\mathrm{gs}}$ to negative $V_{\mathrm{gs}}$ values for p-type materials and from zero $V_{\mathrm{gs}}$ to positive $V_{g s}$ for the n-type semiconductors, $\mathrm{C}_{60}$ ), a fact of significant influence on the OFF level of the drain current, observed also by Horowitz et al. ${ }^{61}$ We went on and measured our devices also in the depletion mode, and observed indeed a tendency of $I_{\text {off }}$ to move towards lower values, as pointed out by
Horowitz et al. This trend is shown by all purity grades of each material and by all materials in this study. It seems plausible to assume then that the change of the morphology of the deposited film due to the increased purity level of the evaporated material generates a film of increased bulk conductivity, which more than offsets the decrease of the $I_{\text {off }}$ level due to the lower impurity content of the film. More studies will be necessary to elucidate this observed event.

Also, it looks obvious that the identical recipe utilized in this work with respect to the semiconductor deposition is not beneficial for all of them in extracting the maximum performance (i.e. charge mobility value or charge mobility balance of the electron and hole channels, as it is the case for Tyrian purple or indanthrene blue RS). For all materials, a specific deposition method has to be implemented for rendering the high performance. ${ }^{52,56,57,60}$ In this respect, several factors need to be carefully considered and optimized for maximum performance of each material in OFETs, like for example: the deposition rate; the substrate temperature during deposition (ideal for the growth of larger crystals which minimizes the number of grain boundaries and the afferent charge trapping 
sites); the distance from the crucible to the substrate holder, the semiconductor layer thickness, etc. As it is visible in Fig. 3 of our already published device, ${ }^{60}$ a thicker layer of $\mathrm{C}_{60}(300 \mathrm{~nm}$ in the respective case), carried out in a very deep vacuum of $\sim 10^{-9}$ bar, at a very low rate of deposition, significantly below $1 \AA \mathrm{s}^{-1}$ and a constant substrate temperature of $150{ }^{\circ} \mathrm{C}$ seems to be an ideal recipe to obtain high semiconductor mobility performance, in spite of the poor transistor geometry of a large channel of $80 \mu \mathrm{m}$ utilized there. It is important to mention that this study focused on showing the trend of improvement in the OFET performance via increasing level of purity of organic semiconductors, and not to engineer record values of charge mobility. This is valid also in our choice of the organic dielectric capping layer, i.e. the thin TTC deposited on top of the aluminum oxide dielectric, which does not afford a substrate heating in excess of $65{ }^{\circ} \mathrm{C}$, whereas typical values of substrate heating in excess of $100{ }^{\circ} \mathrm{C}$ are required for obtaining high mobility semiconductors, as we showed already in one of our previous studies. ${ }^{60}$ Obviously, particular deposition recipes can be made available for each semiconductor to extract high charge carrier mobility, as we proved earlier in the case of epindolidione, ${ }^{56}$ quinacridone, ${ }^{56}$ indigo $^{58}$ and Tyrian purple. ${ }^{52}$ Nevertheless, even though all these factors shall be considered, one will always observe that record values are obtained when the level of cross contamination is minimized, i.e. the vacuum deposition instrument is dedicated for a single material only or cleaned rigorously in-between the change of materials and subsequently utilized for a single material for an extended time. This was indeed the case of $\mathrm{C}_{60}$ deposition in our study in ref. 60, where the organic evaporator instrument was dedicated to handling one material only, i.e. $\mathrm{C}_{60}$ for a very long period of time.

In conclusion, we showed through this study that the purification of organic semiconductors is a critical parameter, resulting in fabricated devices of superior performance. Therefore, we propose that setting a "materials' purity standard" in different labs worldwide will make the results much more reproducible, comparable and competitive. This could bring a leap forward for organic electronics science and technology.

\section{Conflicts of interest}

There are no conflicts to declare.

\section{Acknowledgements}

We gratefully acknowledge the financial support of the Austrian Science Foundation (FWF) within the Wittgenstein Prize for Prof. Sariciftci (Z222-N19).

\section{References}

1 M. Irimia-Vladu, E. D. Glowacki, N. S. Sariciftci and S. Bauer, Green Materials for Electronics, Wiley-VCH, 2018, ISBN: 978-3-527-33865-8.
$2 \mathrm{X}$. Wu, et al., Thermally stable, biocompatible, and flexible organic field-effect transistors and their application in temperature sensing arrays for artificial skin, Adv. Funct. Mater., 2015, 25, 2138-2146.

3 B. Stadlober, M. Zirlk and M. Irimia-Vladu, Route towards sustainable smart sensors: ferroelectric polyvinylidene fluoride-based materials and their integration in flexible electronics, Chem. Soc. Rev., 2019, 48(6), 1787-1825.

4 Handbook of Organic Electronics and Photonics, ed. H. S. Nalwa, ASP Publishers, 2008, ISBN: 1-58883-095-0.

5 Organic Eletronics, ed. H. Klauk, Wiley-VCH, 2006, ISBN: 978-3-527-31264-1.

6 Optoelectronics of Molecules and Polymers, ed. A. Moliton, Springer, Berlin, 2006, ISBN: 0-387-23710-0.

7 Introduction to Organic Electronic and Optoelectronic Materials and Devices, ed. S.-S. Sun and L. R. Dalton, Taylor and Francis, 2008, ISBN: 978-0-8493-9284-9.

8 Flexible Electronics, ed. W. S. Wong and A. Salleo, Springer Berlin, 2009, ISBN: 978-0-387-74362-2.

9 Organic Electronics in Sensors and Biotechnology, ed. R. Shinar and J. Shinar, McGraw Hill, 2009, ISBN: 978-0-07-159675-6.

10 Organic Field Effect Transistors, ed. Z. Bao and J. Locklin, Taylor and Francis, 2007, ISBN: 978-0-8493-8080-8.

11 J. C. Martins and L. A. Sousa, Bioelectronic Vision World Scientific, 2009, ISBN: 978-981-279-430-7.

12 Organic Light Emitting Materials and Devices, ed. Z. Li and H. Meng, Taylor and Francis, 2007, ISBN: 978-1-57444-574-9.

13 Organic Photovoltaics, ed. C. Brabec, V. Dyakonov, J. Parisi and N. S. Sariciftci, Springer Verlag, 2003, ISBN: 3-540-00405.

14 Organic Photovoltaics: Mechanisms, Materials and Devices, ed. S.-S. Sun and N. S. Sariciftci, CRC Press (Taylor \& Francis Group), 2005, ISBN: 0-8247-5963-X.

15 Physics of Organic Semiconductors, ed. W. Brütting, Wiley VCH, 2005, ISBN: 978-3-527-40550-3.

16 L. Sun, Y. A. Diaz-Fernandez, T. A. Gschneidtner, F. Westerlund, S. Lara-Avila and K. Moth-Poulsen, Single-molecule electronics: from chemical design to functional devices, Chem. Soc. Rev., 2014, 43, 7378-7411.

17 A. Facchetti, $\pi$-Conjugated polymers for organic electronics and photovoltaic cell applications, Chem. Mater., 2011, 23, 733-758.

18 V. R. Feig, H. Tran and Z. Bao, Biodegradable polymeric materials in degradable electronic devices, ACS Cent. Sci., 2018, 4(3), 337-348.

19 C. Wang, H. Dong, W. Hu, Y. Liu and D. Zhu, Semiconducting $\pi$-conjugated systems in field-effect transistors: a material odyssey of organic electronics, Chem. Rev., 2012, 112, 2208-2267.

20 J. Mei, Y. Diao, A. L. Appleton, L. Fang and Z. Bao, Integrated materials design of organic semiconductors for fieldeffect transistors, J. Am. Chem. Soc., 2013, 135(18), 6724.

21 H. Sirringhaus, Organic field-effect transistors: the path beyond amorphous silicon, Adv. Mater., 2014, 26, 1319-1335.

22 S. Z. Bisri, C. Piliego, J. Gao and M. A. Loi, Outlook and emerging semiconducting materials for ambipolar transistors, Adv. Mater., 2014, 26, 1176. 
23 P. Bujak, I. Kulszewicz-Bajer, M. Zagorska, V. Maurel, I. Wielgus and A. Pron, Polymers for electronics and spintronics, Chem. Soc. Rev., 2013, 42, 8895-8999.

24 H. Dong, X. Fu, J. Liu, Z. Wang and W. Hu, Key points for high-mobility organic field-effect transistors, Adv. Mater., 2013, 25, 6158-6183.

25 C. Di, F. Zhang and D. Zhu, Multi-functional integration of OFETs: advances and perspectives, Adv. Mater., 2013, 25, 313-330.

26 M. Nikolka, I. Nasrallah, B. Rose, M. K. Ravva, K. Broch, A. Sadhanala, D. Harkin, J. Charmet, M. Hurhangee, A. Brown, S. Illig, P. Too, J. Jongman, I. McCulloch, J.-L. Bredas and H. Sirringhaus, High operational and environmental stability of high-mobility conjugated polymer fieldeffect transistors through the use of molecular additives, Nat. Mater., 2017, 16, 356-362.

27 Organic Electronics, ed. G. Meller and T. Grasser, Springer Berlin, 2010.

28 Semiconducting and Conducting Polymers, ed. A. Heeger, N. S. Sariciftci and E. Namdas, Oxford University Press, 2010.

29 F. C. Grozema and L. D. A. Siebbeles, Mechanism of charge transport in self-organizing organic materials, Int. Rev. Phys. Chem., 2008, 27, 87-138.

30 M. E. Gershenson, V. Podzorov and A. F. Morpurgo, Electronic transport in single-crystal organictransistors, Rev. Mod. Phys., 2006, 78, 973-989.

$31 \mathrm{H}$. Klauk, Will we see gigahertz organic transistors?, Adv. Electron. Mater., 2018, 4(10), 1700474.

32 J. Zaumseil and H. Sirringhaus, Electron and ambipolar transport in organic field-effect transistors, Chem. Rev., 2007, 107, 1296-1323.

33 M. Irimia-Vladu and J. W. Fergus, Impedance spectroscopy of thin films of emeraldine base polyaniline and its implications for chemical sensing, Synth. Met., 2006, 156(21-24), 1396-1400.

34 E. F. Gomez, V. Venkatraman, J. G. Grote and A. J. Steckl, DNA bases thymine and adenine in bio-organic light emitting diodes, Sci. Rep., 2014, 4, 7105.

35 U. Zschieschang, T. Yamamoto, K. Takimiya, H. Kuwabara, M. Ikeda, T. Sekitani, T. Someya and H. Klauk, Organic electronics on banknotes, Adv. Mater., 2011, 23, 654-658.

36 T. R. Ray, J. Choi, A. J. Bandodkar, S. Krishnan, P. Gutruf, L. Tian, R. Ghaffari and J. A. Rogers, Bio-integrated wearable systems: a comprehensive review, Chem. Rev., 2019, 119(8), 5461-5533.

37 R. W. I. de Boer, M. E. Gershenson, A. F. Morpurgo and V. Podzorov, Organic single-crystal field-effect transistors, Phys. Status Solidi A, 2004, 201(6), 1302-1331.

38 N. Karl, K.-H. Kraft, J. Marktanner, M. Münch, F. Schatz, R. Stehle and H.-M. Uhde, Fast electronic transport in organic molecular solids?, J. Vac. Sci. Technol., A, 1999, 17, 2318.

39 A. R. McGhie, A. F. Garito and A. J. Heeger, A gradient sublimer for purification and crystal growth of organic donor and acceptor molecules, J. Cryst. Growth, 1974, 22, 295-297.
40 H. J. Wagner, R. O. Loutfy and C.-K. Hsiao, Purification and characterization of phthalocyanines, J. Mater. Sci., 1982, 17, 2781-2791.

41 C. D. Dimitrakopoulos, A. R. Brown and A. Pomp, Molecular beam deposited thin films of pentacene for organic field effect transistor applications, J. Appl. Phys., 1996, 80, 2501.

42 Y.-Y. Lin, D. J. Gundlach, S. F. Nelson and Th. N. Jackson, Pentacene-based organic thin-film transistors, IEEE Trans. Electron Devices, 1997, 44(8), 1325-1331.

43 R. A. Laudise, Ch Kloc, P. G. Simpkins and T. Siegrist, Physical vapor growth of organic semiconductors, J. Cryst. Growth, 1998, 187, 449-454.

44 J. Mizuguchi, An improved method for purification of copper phthalocyanine, Cryst. Res. Technol., 1981, 16, 695-700.

45 G. Voss and H. Gerlach, Regioselektiver brom/lithiumAustausch bei 2,5-dibrom-1-nitrobenzol. - Eine einfache synthese von 4-Brom-2-nitrobenzaldehyd und 6,6'-Dibromindigo, Chem. Ber., 1989, 122, 1199-1201.

46 M. Irimia-Vladu, Y. Kanbur, F. Camaioni, C. Yumusak, A. A. Vlad, C. V. Irimia, A. Operamolla, G. Farinola, G. Romanazzi, G. P. Suranna, N. González, M. C. Molina, L. F. Bautista, H. Langhals, E. D. Glowacki and N. S. Sariciftci, Stability of selected hydrogen-bonded semiconductors in organic electronic devices, Chem. Mater., 2019, 31(17), 6315-6346.

47 L. A. Majewski, M. Grell, S. D. Ogier and J. Veres, A novel gate insulator for flexible electronics, Org. Electron., 2003, 4, 27-32.

48 M. Irimia-Vladu, P. A. Troshin, M. Reisinger, G. Schwabegger, M. Ullah, R. Schwoediauer, A. Mumyatov, M. Bodea, J. W. Fergus, V. F. Razumov, H. Sitter, S. Bauer and N. S. Sariciftci, Environmentally sustainable organic field effect transistors, Org. Electron., 2010, 11, 1974-1990.

49 A. I. Mardare, M. Kaltenbrunner, N. S. Sariciftci, S. Bauer and A. W. Hassel, Ultra-thin anodic alumina capacitor films for plastic electronics, Phys. Status Solidi A, 2012, 209(5), 813-818.

50 M. Kaltenbrunner, T. Sekitani, J. Reeder, T. Yokota, K. Kuribara, T. Tokuhara, M. Drack, R. Schwödiauer, I. Graz, S. Bauer-Gogonea, S. Bauer and T. Someya, An ultra-lightweight design for imperceptible plastic electronics, Nature, 2013, 499, 458-463.

51 E. D. Glowacki, L. N. Leonat, G. Voss, M. Badea, Z. Bozkurt, M. Irimia-Vladu, S. Bauer and N. S. Sariciftci, Ambipolar organic field effect transistors and inverters with the natural material tyrian purple, AIP Adv., 2011, 1, 042132.

52 Y. Kanbur, M. Irimia-Vladu, E. D. Glowacki, M. Baumgartner, G. Schwabegger, L. N. Leonat, M. Ullah, H. Sitter, R. Schwödiauer, Z. Kücükyavuz, S. Bauer and N. S. Sariciftci, Vacuum processed polyethylene as a dielectric for low voltage operating organic field effect transistors, Org. Electron., 2012, 13, 919-924.

53 M. Irimia-Vladu, N. Marjanovic, M. Bodea, G. HernandezSosa, A. Montaigne Ramil, R. Schwödiauer, S. Bauer, N. S. Sariciftci and F. Nüesch, Small-molecule vacuum processed melamine- $\mathrm{C}_{60}$, organic field-effect transistors, Org. Electron., 2009, 10(3), 408-415. 
54 A. Petritz, A. Fian, E. D. Głowacki, N. S. Sariciftci, B. Stadlober and M. Irimia-Vladu, Ambipolar inverters with natural origin organic materials as gate dielectric and semiconducting layer, Phys. Status Solidi RRL, 2015, 9, 358-361.

55 Y. Kanbur, H. Coskun, E. D. Głowacki, M. Irimia-Vladu, N. S. Sariciftci and C. Yumusak, High temperature stability of organic thin-film transistors based on quinacridone pigments, Org. Electron., 2019, 66, 53-57.

56 E. D. Glowacki, M. Irimia-Vladu, M. Kaltenbrunner, J. Gąsiorowski, M. S. White, G. Romanazzi, G. P. Suranna, P. Mastrorilli, T. Sekitani, S. Bauer, T. Someya, L. Torsi and N. S. Sariciftci, Hydrogen-bonded semiconducting pigments for air-stable field-effect transistors, Adv. Mater., 2013, 25, 1563-1569.

57 A. Petritz, A. Wolfberger, A. Fian, T. Griesser, M. IrimiaVladu and B. Stadlober, Cellulose derivative based gate dielectric for high performance organic complementary inverters, Adv. Mater., 2015, 27, 7645-7656.
58 M. Irimia-Vladu, E. D. Glowacki, P. A. Troshin, D. K. Susarova, O. Krystal, G. Schwabegger, M. Ullah, Y. Kanbur, M. A. Bodea, V. F. Razumov, H. Sitter, S. Bauer and N. S. Sariciftci, Indigo-a natural pigment for high performance ambipolar organic field effect transistors and circuits, Adv. Mater., 2012, 24(3), 375-380.

59 C. R. Newman, C. D. Frisbie, D. A. da Silva Filho, J. L. Bredas, P. C. Ewbank and K. R. Mann, Introduction to organic thin film transistors and design of n-channel organic semiconductors, Chem. Mater., 2004, 16, 4436-4451.

60 G. Schwabegger, M. Ullah, M. Irimia-Vladu, M. Reisinger, Y. Kanbur, R. Ahmed, P. Stadler, S. Bauer, N. S. Sariciftci and H. Sitter, High mobility, low voltage operating C60 based n-type organic field effect transistors, Synth. Met., 2011, 161, 2058-2062.

61 G. Horowitz, R. Hajlaoui and F. Kouki, An analytical model for the organic field-effect transistor in the depletion mode. Application to sexithiophene films and single crystals, Eur. Phys. J.: Appl. Phys., 1998, 1, 36-367. 